\title{
The Metabolic Fate of High Density Lipoprotein (HDL) in the Diabetic Rat*
}

\author{
H. Bar-On and S. Eisenberg \\ Lipid Research Laboratory, Department of Medicine B, Hadassah University Hospital, Jerusalem, Israel
}

\begin{abstract}
Summary. Diabetic rats, pre-fed and maintained on a sucrose-rich diet, have marked hyperlipoproteinaemia, with an increase in both very low density (VLDL) and high density lipoproteins (HDL). HDL obtained from both diabetic and non-diabetic rats and labelled with ${ }^{125} \mathrm{I}$ or ${ }^{131} \mathrm{I}$ was injected simultaneously into diabetic and non-diabetic rats. The half life of the two HDL preparations was similar in both diabetic and non-diabetic rats and ranged between 11.4 to 12.0 hours. A-I apolipoprotein had a disappearance rate parallel to the whole HDL, in contrast to the apo-C peptides which had a faster rate of removal. Although the fractional catabolic rate (FCR) of HDL preparations was slower in the diabetic rats, there was a $16 \%$ increase in the calculated synthetic rate (SR) of HDL-protein. These observations could explain the increased plasma HDL levels in the sucrose-fed, streptozotocin-induced diabetic rat.
\end{abstract}

Key words: Sucrose-rich diet, streptozotocin-induced diabetes, high density lipoprotein, apolipoproteins.

The induction of diabetes mellitus by streptozotocin in rats pre-fed and maintained on a sucrose-rich diet produces a marked increase in the plasma levels of various lipids $[1,2]$. These rats represent an unusual animal model of diabetes mellitus in which the plasma concentrations of both very low density (VLDL) and high densitiy (HDL) lipoproteins are elevated.

\footnotetext{
* This work was presented in part at the 12 th Annual Meeting of the European Association for the Study of Diabetes (EASD), Helsinki, Finland, September 1976
}

Studies to elucidate the mechanism underlying the hypertriglyceridaemia in diabetes mellitus have shown a defect in the removal of VLDL and chylomicrons due to decreased activity of plasma and adipose tissue lipoprotein lipase $[3,4]$. The possibility of an inhibitor of the lipoprotein lipase has also been recently considered [5].

In the rat, which has very small quantities of plasma low density lipoprotein (LDL), the major part of the plasma cholesterol-ester is carried by HDL. The rise in HDL-apoprotein in the plasma of our sucrose-fed diabetic rats is of considerable interest and this paper deals with the metabolic fate of HDL-apoprotein in these animals.

\section{Materials and Methods}

\section{Animals and Diet}

Male rats of the Hebrew University strain weighing about $250 \mathrm{~g}$ were fed for three weeks, prior to the induction of diabetes, a semi-purified sucrose-rich diet consisting of $20 \%$ vitamin-free casein, $60 \%$ sucrose, $5 \%$ lard, $1 \%$ vitamin mixture, $4 \%$ salt mixture, $10 \%$ cellulose. Streptozotocin [ $40 \mathrm{mg} / \mathrm{kg}$ body weight] was injected into the tail vein. This dose was preferred to the previously recommended dose of $45 \mathrm{mg} / \mathrm{kg}$ [1] since the higher dose proved lethal to sucrose-fed rats of the Hebrew University strain. Control rats were injected with $0.5 \mathrm{ml}$ of $0.05 \mathrm{M}$ citric acid containing no streptozotocin. Twelve days after injection, both control non-diabetic and diabetic rats were exsanguinated, under light ether anaesthesia, through the abdominal aorta. The blood was collected and disodium EDTA was added to a final concentration of $1 \mathrm{mg} / \mathrm{ml}$. 
Table 1. Plasma levels of glucose, triglyceride, cholesterol HDLprotein and HDL cholesterol in sucrose fed non-diabetic and diabetic rats

\begin{tabular}{llc}
\hline & Non-Diabetic & Diabetic \\
\hline & $\mathrm{mg} / \mathrm{dl}$ & \\
\cline { 2 - 3 } & $182.4 \pm 11.9(10)^{\mathrm{a}}$ & $685.5 \pm 24.0(12)^{\mathrm{a}}$ \\
Glucose & $245.8 \pm 23.9(10)^{\mathrm{a}}$ & $404.1 \pm 76.8(9)^{\mathrm{a}}$ \\
Triglyceride & $54.7 \pm 1.3(10)^{\mathrm{a}}$ & $68.4 \pm 6.9(9)^{\mathrm{a}}$ \\
Cholesterol & $45.4 \pm 3.5(5)^{\mathrm{b}}$ & $62.6 \pm 4.0(5)^{\mathrm{b}}$ \\
HDL-protein & $40.3 \pm 4.2(5)^{\mathrm{b}}$ & $55.0 \pm 4.2(5)^{\mathrm{b}}$ \\
\hline
\end{tabular}

Values are mean $\pm \mathrm{S}$. E. Numbers in parentheses represent either numbers of animals ${ }^{\mathrm{a}}$ or numbers of pools, each consisting of 2 animals $^{\mathrm{b}}$

Table 2. Percent distribution of radioactivity among various plasma lipoprotein classes in rats following injection of labelled rat HDL

\begin{tabular}{cccc}
\hline $\begin{array}{l}\text { Time } \\
\text { (hours) }\end{array}$ & $\mathrm{d}<1.063$ & $\mathrm{~d}=1.063-1.21$ & $\mathrm{~d}>1.21$ \\
\hline $1 / 12$ & 16.8 & 77.5 & 5.7 \\
1 & 8.2 & 88.3 & 3.5 \\
4 & 7.3 & 87.3 & 5.4 \\
8 & 7.5 & 87.6 & 4.9 \\
24 & 7.2 & 87.0 & 5.8 \\
48 & 10.1 & 73.8 & 16.1 \\
\hline
\end{tabular}

Preparation of Lipoproteins and Labelled Lipoproteins

High density lipoprotein was separated by ultracentrifugation at $\mathrm{d}=1.085-1.21$, as described by Havel et al. [6]. The HDL was resuspended in $\mathrm{KBr}$ $\mathrm{d}=1.21$ solution and recentrifuged. Radiolabelling of HDL with ${ }^{125} \mathrm{I}$ or ${ }^{131} \mathrm{I}$ was performed by a modification of the iodine monochloride method of MacFarlane [7]. The efficiency of iodination was about $50 \%$. Care was taken to obtain a molar ratio of $\mathrm{I}_{2} /$ protein $<1$, assuming a mean molecular weight of HDL apoproteins of 25,000. In some experiments, HDL of diabetic rats was labelled with ${ }^{125} \mathrm{I}$ and that of the non-diabetic rats with ${ }^{131} \mathrm{I}$. In other experiments, the labels were reversed. The pooled HDL was exhaustively dialyzed against $150 \mathrm{mmol} / \mathrm{l}$ sodium chloride to remove the free iodine. After dialysis, more than $95 \%$ of radioactivity was precipitable with $10 \%$ TCA. The radioiodinated HDL isolated from either diabetic or nondiabetic rats on the sucrose-rich diet contained less than $5 \%$ of the total radioactivity in the lipid moiety.

\section{Experimental Design}

Diabetic and non-diabetic sucrose-fed rats were injected with labelled HDL $(0.1-0.2 \mathrm{mg}$ HDL pro- tein, 5-10 $\times 10^{6} \mathrm{cpm}$ ) under light ether anaesthesia through the tail vein. In the experiment, non-diabetic and diabetic rats were injected with the labelled HDL of both groups (diabetic and non-diabetic - see above) and the rats were sacrificed by exsanguination 5 min or $1,4,8,24,48$ hours after the injection. This same experiment was repeated three times. In each experiment two rats were sacrificed at each time interval, and the plasma radioactivity decay was determined. The plasma from the two rats was thereafter combined and used for separation of lipoproteins. Very low density lipoprotein (VLDL) was isolated at $\mathrm{d}<1.006$, low density lipoprotein (LDL) at d 1.006-1.063 and high density lipoprotein (HDL) at $d$ 1.063-1.21. As it was observed in the first experiment that the LDL fraction contained only $1-2 \%$ of the plasma radioactivity, this fraction was not further separated in subsequent experiments from the VLDL. Each fraction was dialysed against $0.9 \%$ sodium chloride solution, containing $0.01 \%$ disodium EDTA at $\mathrm{pH}$ 7.4. Radioactivity was measured in each of the isolated fractions. Apoproteins were separated by SDS polyacrylamide gel electrophoresis using $12.5 \%$ polyacrylamide gels [8]. Protein bands were stained with $0.05 \%$ Coomassie Blue, destained in $7 \%$ acetic acid, sliced by hand and counted.

\section{Biochemical Analysis}

Glucose levels were determined by the Worthington Glucostat Reagent Kit method. Plasma and lipoprotein lipids were extracted in isopropanol and triglyceride and cholesterol levels were determined by the Technicon Auto-analyzer II [9]. Lipoprotein protein was determined by a modification [10] of the Lowry method [11]. Radioactivity was determined using a Packard Auto-Gamma spectrometer (model No. 578).

\section{Results}

Twelve days after the administration of streptozotocin to sucrose-fed rats, diabetes mellitus is well established and is accompanied by hyperlipaemia and hyperlipoproteinaemia (Table 1). Following injection of labelled HDL into both groups of rats, more than $70 \%$ of the radioactivity recovered at all time intervals was in the HDL fraction (Table 2).

The decay of labelled HDL from the plasma was bi-exponential in the two groups of rats and with both HDL preparations. The decay curves of labelled HDL, isolated from both non-diabetic and diabetic rats, are shown in Figure 1. The HDL dis- 


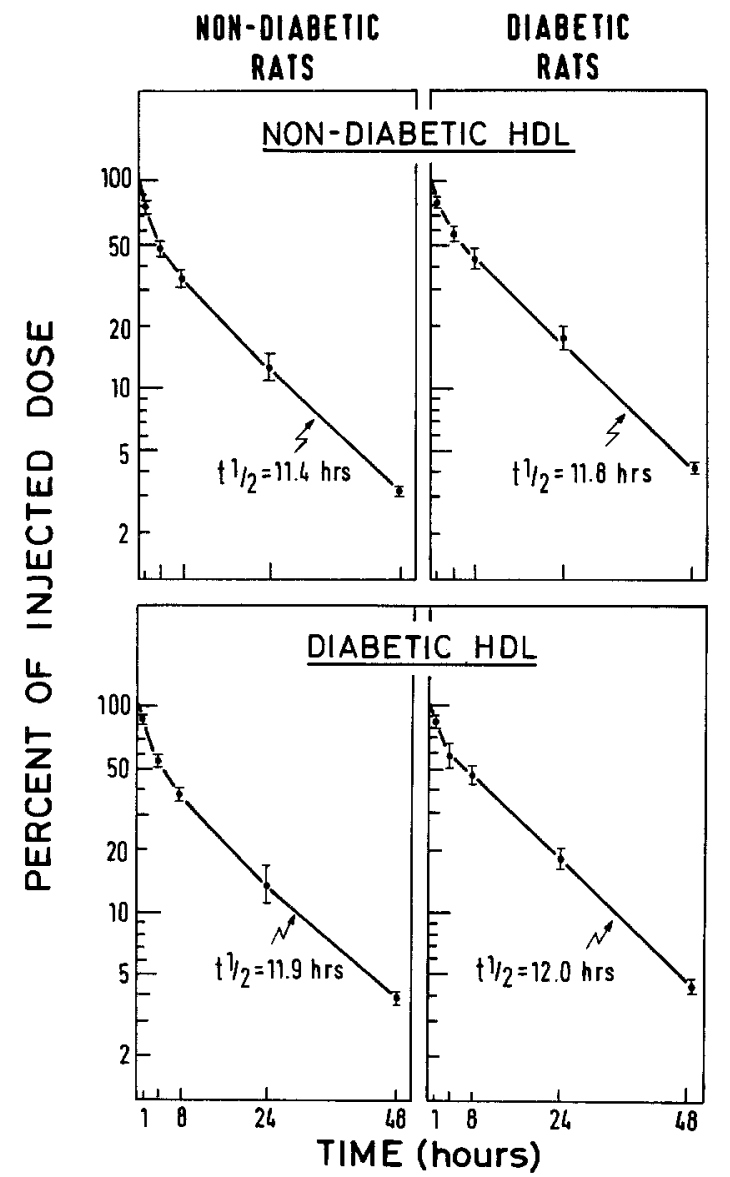

Fig. 1. Plasma decay curves of labelled whole high density lipoprotein (HDL) in non-diabetic and diabetic rats

appeared from the blood of both groups at a similar rate. The half-life of the second component of the HDL decay curves ranged from 11.4-12.0 hours.

Since HDL of diabetic rats differs in its apolipoprotein composition from that of normal rats, the decay curves of individual apoproteins were determined by SDS polyacrylamide gel electrophoresis. Figure 2 shows a typical distribution of non-diabetic and diabetic apo-HDL pattern. The diabetic HDL has much less arginine-rich peptide (ARP) and less A-IV peptide than non-diabetic HDL. The gels were stained and sliced and the individual bands were counted except for the apo-C peptides that were treated as one band. Figure 3 shows plasma decay radioactivity curves of whole HDL, A-I, apo-C peptides and ARP. In all experiments, the rate of removal of A-I was parallel to the decay curve of whole HDL. The disappearance rates of apo-C peptides and ARP were faster than those of A-I and whole HDL. The removal rate of apo-C peptides in the diabetic rats was slower than in the non-diabetic rats.

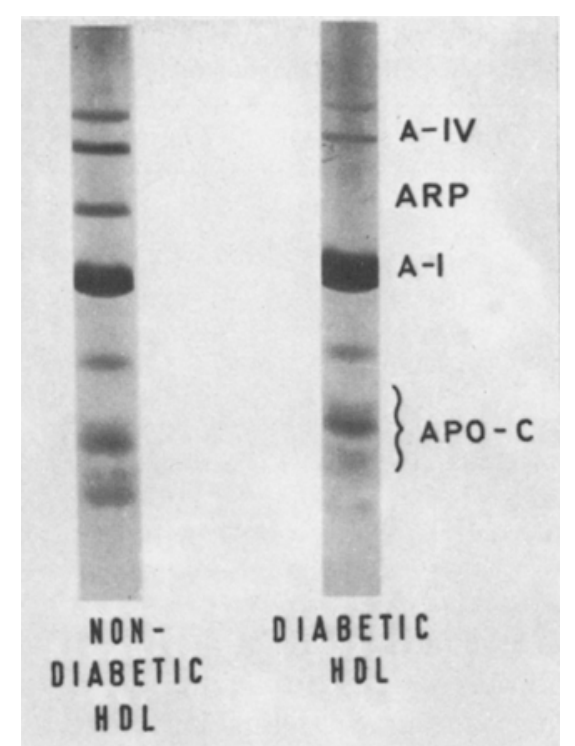

Fig. 2. SDS-Polyacrylamide gel electrophoretic distribution of apolipoproteins of non-diabetic and diabetic HDL

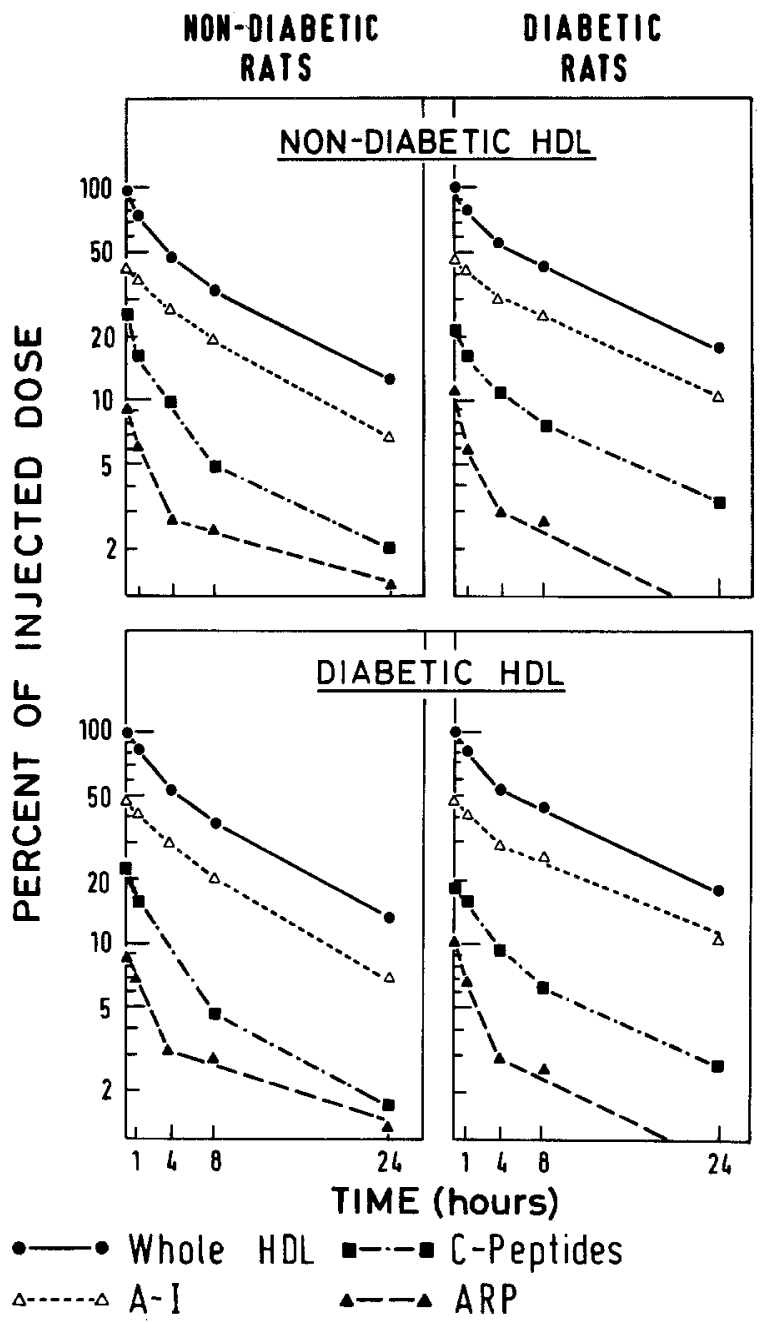

Fig. 3. Plasma decay curves of labelled HDL-apolipoproteins in non-diabetic and diabetic rats 
Table 3. Fractional catabolic rates (FCR) and synthetic rates (SR) of whole HDL in non-diabetic and diabetic rats

\begin{tabular}{llcc}
\hline & Non-diabetic rat & Diabetic rat \\
\hline HDL & $\begin{array}{l}\text { Protein-pool } \\
\text { mg/dl }\end{array}$ & 45.4 & \\
FCR- & N HDL & 0.1017 & 62.6 \\
& D HDL & 0.0898 & 0.0817 \\
& mean & 0.0957 & 0.0801 \\
SR & N HDL & 4.6160 & 0.0809 \\
& D HDL & 4.0760 & 5.1144 \\
& mean & 4.3460 & 5.0149 \\
\hline
\end{tabular}

a N HDL - Non-diabetic HDL

b D HDL - Diabetic HDL

Fractional catabolic rates (FCR) of the two HDL preparations in non-diabetic and diabetic rats were calculated following the curve peeling technique as described by Matthews for steady-state conditions [12]. Synthetic rates were obtained by multiplication of the FCR by the HDL protein pool size. Since there were minor weight differences of the animals in the two groups studied, the HDL pool sizes were calculated as the amount of HDL-protein per $100 \mathrm{ml}$ serum.

The fractional catabolic rate (FCR) and the synthetic rate (SR) of HDL apo-proteins in nondiabetic and diabetic rats are shown in Table 3. Although the fractional catabolic rate of HDL apoprotein in non-diabetic rats was higher than in diabetic rats, the calculated synthetic rate was higher in the diabetic rats by an average of $16 \%$.

\section{Discussion}

The objective of the present investigation was to study the metabolism of high density lipoprotein apoprotein in streptozotocin-induced sucrose-fed diabetic rats. The simultaneous injection of differently labelled HDL isolated from diabetic and nondiabetic rats enabled us to compare the metabolic fate of the two different HDL preparations in both diabetic and non-diabetic groups. No marked differences in the removal rate were found between the two HDL preparations.

This observation suggested that in spite of the known differences of apoprotein pattern of diabetic and non-diabetic HDL [2] the rats have not distinguished between the two lipoprotein particles. With both preparations, in diabetic and non-diabetic rats, the HDL decay curve was bi-exponential, similar to the decay curve of normal HDL in normal rats of the Hebrew University strain [13]. The half-life of the HDL in the circulation was 11.4-12.0 hours, slightly longer than the 10.5 hours reported by Roheim et al. [13], but in good agreement with the findings of Eisenberg et al. [14]. In spite of the use of different diets in each of these studies there was little difference in HDL kinetics.

The decay of HDL apoproteins was determined after separation by SDS polyacrylamide gel electrophoresis. Most of the HDL radioactivity was found associated with apo-A-I and apo-C. The decay of radioactivity of apo-A-I was almost parallel to that of total HDL radioactivity, with both HDL preparations and in diabetic and non-diabetic rats. Labelled apo-C peptides disappeared from the HDL density range more rapidly than apo-A-I. They were transferred to VLDL where iodinated apo-C contributed $50-75 \%$ of the total VLDL radioactivity. Similar results have been previously reported [13, 14]. However, in diabetic rats their subsequent decay from HDL was slower than in non-diabetic rats, presumably reflecting the larger apo-C exchangeable pool of the high VLDL levels of these animals [2].

Kinetic analysis of HDL metabolism in sucrosefed diabetic and non-diabetic rats was performed using parameters measured for the whole lipoprotein. Since apo-A-I is the major apo-protein of HDL in both groups of rats and its decay paralleled that of whole HDL, these calculations most probably reflect predominantly the metabolism of apo-A-I. Two methods were employed to calculate fractional catabolic rates and synthetic rates. The first method followed the bi-exponential analysis as suggested by Matthews [12]. The second method assumed only one true decay curve, that of the slower component. With both methods we found that the fractional catabolic rate of both HDL preparations in diabetic rats was slower than in non-diabetic rats and that the synthetic rate was higher. Although the primary defect is not apparent from the present study it is tempting to assume that an initial increase of HDL synthesis results in saturation of removal sites and a subsequent decreased fractional catabolic rate. This hypothesis is supported by recent unpublished observations in our laboratory showing increased secretion of HDL in the isolated perfused liver of diabetic rats as compared to non-diabetic rats.

HDL kinetics in humans indicate an interesting reciprocal relationship between plasma triglyceride levels and HDL removal rates. With carbohydrate feeding when plasma TG levels increase and HDL levels decrease the fractional catabolic rate of HDL increases. However, with nicotinic acid treatment, plasma TG levels decrease, HDL levels increase and the fractional catabolic rate of HDL decreases. The 
present model is another example of a study of HDL kinetics where both plasma VLDL and HDL levels are affected. However, whereas in the human, VLDL and HDL levels appear to be reciprocally related, in the diabetic rat both are increased. Accordingly, the metabolic events leading to higher $\mathrm{HDL}$ levels may be different and related to increased synthesis rather than decreased catabolism. It is yet unknown whether this mechanism of increased HDL levels may be related to the recently described defect in VLDL removal in diabetic rats [16].

Acknowledgements. This work was supported by a grant 604 from the United States-Israel Binational Foundation (BSF), Jerusalem, Israel. The skilful help of Miss Daniella Landau is gratefully acknowledged. The authors wish to thank Prof. Gershom Zaijcek for his help with the statistical analysis.

\section{References}

1. Bar-On, H., Roheim, P.S., Eder, H.A.: Hyperlipoproteinaemia in streptozotocin-treated rats. Diabetes $\mathbf{2 5}$, 509-515 (1976)

2. Bar-On, H., Roheim, P.S., Eder, H.A.: Serum lipoproteins and apolipoproteins in rats with streptozotocin-induced diabetes. J. Clin. Invest. 57, 714-721 (1976)

3. Bierman, E. L., Amaral, A.P., Belknap, B. H.: Hyperlipemia and diabetes mellitus. Diabetes 15, 675-679 (1966)

4. Schnatz, D.J., Williams, R. H.: The effect of acute insulin deficiency in the rat on adipose tissue lipolytic activity and plasma lipids. Diabetes 12, 174-178 (1963)

5. Bar-On, H., Roheim, P.S., Eder, H. A.: Apolipoprotein composition and lipoprotein lipase activation in experimental diabetes mellitus. J. Clin. Invest. 58, 4 a (Abstract) (1974)

6. Havel, R. J., Eder, H.A., Bragdon, J.H.: The distribution and chemical composition of ultracentrifugally separated lipoproteins in human serum. J. Clin. Invest. 34, 1345-1353 (1955)
7. MacFarlane, A.S.: Efficient trace-labelling of proteins with iodine. Nature 182, 53 (1958)

8. Maizel, J.V.Jr.: Polyacrylamide gel electrophoresis of viral proteins. Methods Virol. 5, 179-246 (1971)

9. Manual of Laboratory Operations. Lipid Research Clinics Program, Vol. I, Lipid and lipoprotein analysis. Bethesda, Maryland: National Heart and Lung Institute, N. I. H. 1974

10. Sata, T., Havel, R. J., Jones, A. L.: Characterization of subfractions of triglyceride-rich lipoproteins separated by gel chromatography from blood plasma of normolipemic and hyperlipemic humans. J. Lipid Res. 13, 757-768 (1972)

11. Lowry, D., Rosebrough, N. J., Farr, A. L., Randall, R. J. Protein measurement with the Folin phenol reagent. J. Biol. Chem. 193, 265-275 (1951)

12. Matthews, C.M.E.: The theory of tracer experiments with ${ }^{131}$ I plasma proteins. Phys. Med. Biol. 2, 36-53 (1957)

13. Roheim, P.S., Rachmilewitz, D., Stein, O., Stein, Y.: Metabolism of iodinated high density lipoproteins in the rat. I. Half-life in the circulation and uptake by organs. Biochim. Biophys. Acta 248, 315-329 (1971)

14. Eisenberg, S., Windmueller, H. G., Levy, R. I.: Metabolic fate of rat and human lipoprotein apoprotein in the rat. J. Lipid Res. 14, 446-458 (1973)

15. Blum, C. B., Levy, R. I., Hall, M., Goebel, R., Berman, M.: Reciprocal changes in high density lipoprotein metabolism with nicotinic acid treatment and carbohydrate feeding. Circulation 53-54, (Suppl. II) 26 (1976)

16. Van Tol, A.: Hypertriglyceridemia in the diabetic rat. Defective removal of serum very low density lipoproteins. Atherosclerosis 26, 117-128 (1977)

Received: May 12, 1977,

and in revised form: September 1, 1977

H. Bar-On, M. D.

Department of Medicine B

Hadassah University Hospital

P. O. Box 499

Jerusalem

Israel 